\title{
OBJETOS Y PROPIEDADES
}

\section{Anastasio ALEMÁN}

Uno de esos contumaces problemas de la filosofía lo constituye el problema ontológico concerniente a la existencia de objetos y propiedades. No en vano resulta posible retrotraer los orígenes del problema a la controversia entre Platón y Aristóteles en torno a la existencia de universales. $Y$ cuando se menciona este último problema la en ocasiones olvidada filosofía medieval vuelve a recobrar una cierta actualidad. Sin embargo no es mi propósito en estas páginas examinar explícitamente los antecedentes históricos de nuestro problema, aunque esto no signifique tampoco que prescindamos completamente de ellos; tal cosa no sería deseable, aún si fuera posible (y no lo es). La presencia de los antecedentes históricos se encontrará aquí del modo en el que se hace presente lo auténticamente clásico: impregnando inevitablemente nuestro propio discurso. No resultará difícil apreciar las afinidades entre lo que aquí defendemos y parte de las posiciones aristotélicas, aunque se evite el compromiso con el realismo ingenuo comúnmente atribuido al estagirita, con mejores o peores razones. $\mathrm{Al}$ decir esto último lo que pretendo indicar es que lo que aquí se defiende resulta igualmente compatible, y quizá con mayor afinidad, con una interpretación del problema de corte kantiano.

Pero sea esto como sea, lo cierto es que el origen de mis presentes reflexiones sobre el problema no arrancan de las anteriores posiciones mencionadas, sino de uno de los intentos contemporáneos más lúcidos por clarificarlo. Me refiero al análisis de Quine sobre el denominado "importe ontológico" de una teoría.

Éndoxa: Series Filosóficas, $n^{\circ} 9,1997$, UNED, Madrid:

Anastasio Alemán: Objetos y propiedades.

pp. 101-123. 
Como es bien sabido entre los resultados de ese tipo de análisis se encuentra la tesis de que aunque objetos físicos y clases son tipos de entidades cuya existencia no resulta problemática, sin embargo no se encuentran en análoga situación las propiedades. Estas últimas pertenecen, si se me permite hablar así, a la categoría de las entidades sospechosas. Y el origen de la sospecha radica, en opinión de Quine, en su carencia de un principio de identificación, o individuación, análogo al disponible para objetos físicos y clases. La consecuencia dramática (jen este contexto!) de tal carencia es que la propia existencia de propiedades queda, cuanto menos, en entredicho.

I. Nuestro problema inmediato consiste pues en intentar decidir si existen o no propiedades. Mas ¿Cómo abordar esta cuestión?, ¿Qué criterio emplear para determinar una respuesta positiva o negativa a la pregunta acerca de la existencia de propiedades?

Hay un célebre criterio que se ha ofrecido con el propósito de determinar el importe óntico de una teoría. Se trata del conocido criterio ontológico de Quine.

De entre las múltiples formulaciones que Quine ha ofrecido de este criterio quizás la más conspicua para nuestros fines sea aquella que apela a lo que satisface a los predicados de la teoría: "Otro modo de decir qué objetos requiere una teoría es decir que hay aquellos objetos de los que algunos predicados de la teoria han de ser verdaderos para que la teoría sea verdadera" [OR, $p$. 95]. Esta formulación del criterio la considera equivalente a la más conocida "ser es ser el valor de una variable" como se pone explícitamente de manifiesto cuando nos dice que "En vez de decir ser es ser el valor de una variable' podemos aún decir igualmente 'ser es ser denotado por un término general', eso es, 'ser es satisfacer a un predicado"' (Respuesta a Blasco p. 165).

Como es bien sabido el criterio de Quine no nos dice directamente qué es lo que hay, sino lo que una teoría dice o implica que hay. Lo que hay es lo que una teoría verdadera dice que hay. Es 
decir el criterio ontológico no pretende decir qué es lo que hay o existe en el mundo, esta es una tarea para la ciencia, sino determinar qué se sigue que hay a partir del supuesto de que determinada teoría fuera verdadera. Así entendido, al determinar el importe óntico de una teoría no nos comprometemos con los compromisos ónticos implícitos en la teoría como algunos críticos han objetado (por ejemplo Sheffler y Chomsky) pues lo que establecemos aplicando el criterio es un condicional cuyo antecedente es la tesis, o supuesto, de la verdad de la teoría, y cuyo consiguiente es el importe óntico de la teoría. Pero como es obvio la aceptación del condicional no implica la aceptación de los compromisos ónticos de la teoría que aparecen en su consecuente. Para que hubiera tal implicación se requeriría la aceptación del antecedente, esto es de la verdad de la teoría, pero esto es algo de lo que podemos prescindir cuando nuestro objetivo es meramente, pero nada menos, que determinar el importe óntico de una teoría.

II. Sin embargo una objeción más seria al criterio de Quine sería que éste resulta excesivamente parsimonioso respecto a las implicaciones ónticas que habría que extraer, de acuerdo con el criterio, del supuesto de la verdad de determinado trozo de discurso. Un ejemplo nos permitirá ilustrar más claramente este punto.

Así supongamos la verdad del enunciado:

(1) Sócrates es valiente 
Según el análisis de Quine la verdad de (1) sólo implica la existencia de Sócrates y, en mi opinión, esto es un error. La verdad de (1) implica ciertamente la existencia de Sócrates, pero no sólo implica eso. Implica también la existencia, en su momento, de cierto tipo de acciones realizadas por Sócrates que han de ser cualificadas o caracterizadas como valientes.

La verdad de este último aserto no es difícil de probar: $\mathrm{Si}$ Sócrates no existió entonces (1) no sería verdadero (como hemos supuesto), y esto no parece requerir mayor argumentación. Luego si (1) es verdadero entonces, por implicación, Sócrates existió. Pero no menos claro resulta que aún si Sócrates existió pero no realizó el tipo de acciones que cabe describir correctamente como valientes entonces sería falso decir que Sócrates fue valiente (en abierta contradicción con nuestro supuesto de partida).

En resumen, la verdad de (1) no sólo implica la existencia de Sócrates sino también la existencia de cierto tipo de acciones realizadas por Sócrates.'

III. Quizás se sienta la tentación de formular una objeción aparentemente obvia:

El criterio de Quine sólo pretende indicar qué objetos se presupone, o se implica, que hay y las acciones no son objetos.

El análisis anterior puede considerarse como el cumplimiento, largamente demorado, de una promesa implicita, e incumplida, en mi libro sobre las categorías cuando hablaba de las discrepancias entre Strawson y Quine a propósito del importe óntico del enunciado (1). Sin embargo, como resulta fácil de apreciar, tal cumplimiento no ha seguido exactamente la línea que allí se sugería. 
El criterio detecta correctamente los objetos involucrados, así que no se puede apelar a la presunta existencia de acciones para criticar el criterio de compromiso óntico propuesto por Quine.

Pero a esto cabe responder que si admitimos que la verdad de (1) implica la existencia de objetos, -Sócrates-y, de acciones -que muestren valentía- y el criterio de Quine sólo permite 'detectar' la existencia de objetos, entonces tal criterio no permite detectar todo lo que existe, todos los tipos de entidades implicadas por la verdad de los enunciados que aceptamos hipotéticamente como verdaderos. Y esto es precisamente lo que buscamos: desvelar todas las implicaciones ónticas de nuestro discurso.

Es precisamente en ese sentido en el que decimos que la verdad de 'Sócrates es valiente' implica tanto la existencia de Sócrates como la existencia de acciones de cierto tipo. $Y$ aquí no cabe objetar, o al menos no cabe hacerlo desde una perspectiva quiniana, que la atribución de existencia a acciones es un asunto más obscuro que su atribución a objetos físicos como Sócrates, pues ha sido el propio Quine quien con más vigor ha insistido en que 'existe' se dice en el mismo sentido de objetos físicos que de números y conjuntos, y no parece que las acciones sean entidades ontológicamente más dudosas que los números por ejemplo. De las acciones, $o$ al menos de algunas de ellas, tiene perfecto sentido localizarlas y fecharlas. Así por ejemplo, el conjunto acciones describibles como 'explicaciones del presidente del gobiemo Felipe González a los diputados en torno a las escuchas del CESID pueden fecharse (el 29 de Junio de 1995) y localizarse (en el Congreso de los Diputados), cosa que no tiene sentido hacer con las entidades matemáticas a las que Quine no encuentra reparo alguno en atribuirles existencia.

Negar la existencia de acciones conduce al absurdo de negar que lo que un juez investiga es si hubo o no hubo ciertas acciones realizadas por determinado agente, y abstenerse de afirmar su 
existencia conduce a dejar inexplicado lo que hacemos cada día. Desde luego no pretendo sugerir con esto que Quine esté negando la existencia de acciones; pero lo que sí pretendía mostrar es que éste no aprecia que la verdad de enunciados como (1) no sólo implica la existencia de objetos físicos como Sócrates sino también la existencia de acciones del tipo de las acciones valientes.

IV. La parsimonia del criterio de Quine se muestra más claramente aún si cabe en el análisis de enunciados como:

(2) Velázquez es (fue) un pintor

pues de acuerdo con el criterio que criticamos la verdad de (2) sólo implica la existencia del objeto físico Velázquez; pero parece claro que si (2) es verdadero entonces tiene que haber (o haber habido) algo que Velázquez pintara. No diríamos de nadie que es pintor si no hay al menos algo que haya sido pintado por él. Es decir, ' $x$ es pintor' implica ' $\exists y$ x pintó $y$ ', $y$ esta implicación no es una implicación lógica en el sentido quiniano, esto es, no es una implicación debida a las reglas de uso de las constantes lógicas. Se trata de una implicación semántica debida al significado, uso, del predicado ' $x$ es pintor'. Se trata de lo que Carnap denominaba 'postulados de significado' (p. 222ss.) y que siempre han sido tan denostados por Quine. 
Ciertamente el propio Quine no se priva de realizar este tipo de inferencias que apelan, implícitamente, a tal tipo de postulados; así cuando afirma que al decir que hay, o existen, cisnes negros, decimos por implicación que hay, o existen, objetos físicos [ WP, 205 J. Como es obvio la implicación aquí no es puramente lógica si por tal entendemos condicional lógicamente verdadero. Es decir, la premisa implicita ' $\forall x$ ( $x$ es un cisne negro $\rightarrow x$ es un objeto físico)' no es una verdad lógica, sino un ejemplo paradigmático de lo que solemos entender por 'postulado de significado'.

Lo que el análisis precedente pone de manifiesto es que para desvelar las implicaciones ónticas de enunciados como (2) necesitamos apelar a postulados de significado implícitos en nuestro uso de los términos y que si estos no se reconocen, o negaremos en la teoría lo que reconocemos en la práctica, o pasaremos por alto algunos de los supuestos ónticos de nuestro discurso.

V. Efectivamente, es el rechazo o, al menos, el no reconocimiento de los postulados de significado lo que ha conducido, en $\mathrm{mi}$ opinión, a no reconocer que la existencia de propiedades está implicada en la admisión de la verdad de ciertos enunciados formalizables de modo natural en lógica de primer orden.

Uno de tales postulados de significado es el siguiente:

PS. Si la propiedad $P$ no existe entonces ' $\exists x P x$ ' es falso 
La importancia de este postulado resulta crucial para nuestro objetivo de probar la existencia de propiedades a la par con la existencia de objetos físicos ordinarios, pues de PS junto con la admisión de la verdad de un enunciado de la simple forma ' $\exists x$ $\mathrm{Px}^{\prime}$ se deduce por modus tollens la existencia de propiedades 0 , al menos, la existencia de una propiedad.

VI. Ahora bien ¿Hemos de admitir PS? ¿Hay algún modo de 'probarlo'? Lo que voy a intentar es una prueba informal por reducción al absurdo, esto es, voy a intentar mostrar que el intento de suponer que PS es falso conduce a contradicción.

Así pues supongamos que PS es falso. Si PS es falso esto quiere decir que su antecedente es verdadero y su consiguiente falso. Pero decir que el consiguiente es falso resulta equivalente a decir, en lógica bivalente, que ' $\exists \times \mathrm{Px}^{\prime}$ ' es verdadero; y decir que ' $\exists \times \mathrm{Px}^{\prime}$ es verdadero equivale a decir que la función enunciativa ' $P x^{\prime}$ ' es satisfecha por al menos un objeto.

Por otra parte si PS es falso hemos de admitir la verdad de su antecedente, mas ¿qué significa 'la propiedad P no existe'? Aquí parece haber dos posibilidades de interpretar esta expresión; la segunda es más fuerte que la primera:

En un primer sentido al negar la existencia de una cierta propiedad estamos diciendo que tal propiedad carece de instancias, esto es, al decir 'La propiedad P no existe' estamos diciendo algo equivalente a 'La propiedad P carece de instancias'. Por ejemplo, la propiedad de ser un atleta que ha recorrido cien metros en menos de ocho segundos, carece de instancias. Lo que 
tenemos aquí es un predicado significativo 'es un atleta....' que no se aplica con verdad a ningún objeto y en este sentido decimos que tal predicado no expresa realmente una propiedad existente. Así decir que la propiedad $\mathrm{P}$ carece de instancias es tanto como decir que no hay ningún objeto que satisfaga la función enunciativa ' $\mathrm{Px}$ ', y esto último está en contradicción manifiesta con lo que hemos deducido antes del supuesto de la falsedad del consiguiente de PS, a saber, que la función enunciativa 'Px' es satisfecha por al menos un objeto.

Un segundo modo de interpretar la expresión 'La propiedad P no existe' consiste en considerarla equivalente a la expresión 'No puede haber objeto alguno que constituya una instancia de P'. Esta interpretación es más fuerte que la anterior; pues si no puede haber instancias de la propiedad $P$ entonces esa propiedad carece de instancias. Creo que es en esta acepción en la que solemos decir cosas tales como 'No existe la propiedad de ser un circulo cuadrado'. Mas decir que no puede haber ningún objeto que constituya una instancia de $\mathrm{P}$ es tanto como decir que no puede haber ningún objeto que satisfaga a 'Px', y si no puede ${ }^{2}$ haber ningún objeto que satisfaga a 'Px'entonces, a fortiori, no hay ningún objeto que satisfaga a 'Px', y esto nuevamente está en contradicción directa con lo que deducimos del supuesto de la falsedad del consiguiente de PS.

2 No puede negarse que el termino 'posible', al menos en la forma de 'puede', aparece usado correctamente en multitud de ocasiones aun por los niños en las primeras fases de su aprendizaje del lenguaje. En concreto mi hija Irene ofrecio muestras inequívocas de uso correcto del término 'puede' mucho antes de que fuera capaz de usar correctamente los términos que denotan colores. Así era capaz de decir 'no se puede' ante la imposibilidad de introducir su osito de peluche en una caja de zapatos y de decir 'se puede' cuando lo que introducía era alguno de sus juguetes de menor tamaño. Sin embargo era incapaz a la misma edad (dos años y medio) de usar correctamente los términos de color como 'rojo' o 'blanco' aun cuando mostraba signos inequívocos de que discriminaba entre los colores correspondientes. 
Comprobamos así que el supuesto de que PS es falso conduce a contradicción, luego PS tiene que ser verdadero.

Mas, ¿qué quiere decir que PS es verdadero? En mi opinión PS, a pesar de su forma gramatical enunciativa, lo que expresa realmente es una regla del lenguaje que hablamos. En ocasiones expresamos reglas mediante el uso de oraciones con forma gramatical declarativa, o enunciativa, como se pone especialmente de manifiesto cuando atendemos a la formulación de las reglas del ajedrez; por ejemplo: 'El alfil se desplaza en diagonal' ${ }^{3} \mathrm{La}$ forma gramatical declarativa puede inducir a pensar que lo que estamos expresando es la ocurrencia de determinado estado de cosas; pero si reparamos en cómo usamos realmente la expresión se disipa rápidamente esa impresión inicial; pues el punto clave es que nada contaría como posible contraejemplo a la última oración mencionada; así si el desplazamiento de la pieza no se efectúa en diagonal o no se trata del alfil o lo hemos desplazado incorrectamente. Lo mismo ocurre con el enunciado ' $\forall \mathrm{x}$ ( $\mathrm{x}$ es un cisne negro $\rightarrow \mathrm{x}$ es un objeto físico)' que denominamos anteriormente 'postulado de significado'. De ' $x$ no es un objeto físico' inferimos ' $x$ no es un cisne negro', pero la corrección de esta inferencia depende del postulado mencionado (aunque no sólo depende de él).

Aceptar la corrección de esta última inferencia implica que no aceptamos ningún contraejemplo al postulado de significado empleado tácita, pero esencialmente, en ella. Por ello decimos que

Para más detalles acerca del modo en el que expresamos reglas mediante oraciones declarativas véase mi artículo citado en la bibliografía. 
negar un postulado de significado implica un cambio de significado en alguna de las expresiones que lo formulan. Esto es lo que pone de manifiesto que estamos usando el enunciado como una regla de nuestro lenguaje. Ciertamente podemos negar el enunciado, y con ello cambiar la regla; pero esto equivale a hablar otro lenguaje aunque se sirva de los mismos signos. De nuevo el caso de las reglas de ajedrez resulta paradigmático: cambio de reglas implica cambio de juego (aunque continuemos usando las mismas piezas).

VIII. Hay, aparentemente, una objeción básica a uno de los supuestos tácitos empleados en la argumentación a favor de PS que de tener éxito minaría todo el argumento. La objeción podria formularse brevemente asi: La expresión 'La propiedad P existe' esta mal formada, usa 'existe' como predicado.

Sin embargo en esta objeción subyace un problema previo: ¿Qué criterio se emplea aquí de bien o mal formado? Desde luego no es mal castellano emplear tal expresión, así que el criterio tácito empleado por nuestro hipotético objetor no puede proceder de las reglas de formación de oraciones bien formadas de la gramática del castellano, reglas no completamente formuladas hasta ahora.

¿Quizás se está apelando como criterio a la gramática de la lógica de primer orden? Ciertamente no parece que haya modo adecuado de formalizar 'La propiedad $P$ existe' en el lenguaje de la lógica de primer orden, pero esto no puede constituir la base de una objeción al empleo de tal expresión, pues de lo contrario también habría que objetar el empleo de la familiar expresión ' $\mathrm{Si}$ no hubieses cogido el vaso a tiempo se habría roto irrepara- 
blemente' o 'La economía alemana ha crecido y no ha registrado inflación, luego es posible para una economía crecer sin inflación', o aun 'Napoleón tenía todas las cualidades de un gran general salvo una' (Russell?, Quine).

Considerar que lo que no es formalizable en lógica de primer orden está mal formado o es ininteligible resulta demasiado obviamente falso como para poder fundar una objeción sobre esta premisa. (¿Qué sería entonces de los condicionales subjuntivos empleados por cualquier investigador en sus hipótesis sobre los fenómenos de la naturaleza?)

Mas aun, supongamos por un momento que la expresión 'La propiedad P existe' es una expresión mal formada y que por tanto carece de significado. Nada impide entonces adoptar la convención explícita de tratarla como una paráfrasis equivalente, por ejemplo, a la expresión bien formada " $\exists x$ Px' es verdadero' y dotarla así de significado. No estoy proponiendo aquí tal paráfrasis (aunque exploraremos esta posibilidad a continuación); lo que estoy indicando es que con tal paráfrasis, o con alguna otra similar, se mostraría lo insostenible de la objeción que nos ocupa. Lograríamos clarificar, por la vía de la paráfrasis, una expresión inicialmente problemática mediante otra que, prima facie, lo es menos.

IX. ¿Qué decir de la posibilidad de paráfrasis que acabamos de sugerir? La sugerencia era tratar la expresión 'La propiedad $\mathrm{P}$ 
existe' como equivalente a la expresión ' $\exists$ x Px' es verdadero'. Esta equivalencia resultaría de añadir a PS su conversa: ${ }^{4}$

CPS. Si la propiedad P existe entonces ' $\exists$ x Px' es verdadero.

Lo que CPS viene a decir es que no existen propiedades que carezcan de instancias $o$, dicho de otro modo, que una propiedad no existe si no existe al menos un objeto que tenga tal propiedad. En este sentido diríamos, por ejemplo, que aunque el predicado 'es un atleta que ha recorrido cien metros en menos de ocho segundos' es significativo, no existe, sin embargo, la propiedad correspondiente en tanto en cuanto no hay, aún, ningún atleta que haya recorrido tal distancia en el tiempo señalado. Asimismo podríamos decir que aunque de momento no existe tal propiedad, esta propiedad devendría a la existencia en el preciso momento en el que hubiera un atleta que recorriera los cien metros en menos de ocho segundos. ${ }^{5}$

4 La equivalencia se aprecia más claramente si formulamos PS en su forma contrapuesta: $\mathrm{Si}$ ' $\exists x P x$ ' es verdadero entonces la propiedad $P$ existe.

5 D.M. Armstrong, entre otros, no estarfa de acuerdo con que las propiedades puedan devenir temporalmente a la existencia como lo hacen los objetos físicos, pero también afirma que "Esto no significa que sean objetos platónicos eternos" (p. 158). No sé como podrían conciliarse estas dos afirmaciones, sobre todo teniendo en cuenta que para él, como para mi, las propiedades no son nada sin los objetos que las sustentan. 
Pese a la fuerte plausibilidad de estas observaciones, creo sin embargo que el estatus semántico de CPS resulta diferente del de PS en un aspecto crucial.

Así mientras que la negación de PS conduce a consecuencias absurdas no parece que ocurra lo mismo con la negación de CPS. Al negar PS nos vemos abocados al absurdo de decir que aunque no existe la propiedad $P$ no obstante existe al menos un objeto que tiene tal propiedad, o que aunque 'P' se aplique con verdad a un objeto no existe sin embargo la propiedad, o rasgo, del objeto que hace que resulte verdadera tal predicacion; no parece posible que un objeto tenga propiedades inexistentes.

Sin embargo al negar CPS no aparece ningún absurdo semejante. Alguien podría argüir, por ejemplo, que no hay ningún absurdo en la idea de que existan propiedades aún no instanciadas, ${ }^{6}$ propiedades posibles. En esta concepción cualquier predicado consistente expresaría una propiedad existente. 'Existente' y 'consistente' acabarían como sinónimos, al menos en el contexto de las propiedades. No veo contradicción alguna en esto, pero pese a su plausibilidad en el dominio de discurso de la matemática por ejemplo, no me parece que pueda mantenerse en el dominio de nuestro discurso sobre objetos físicos y sus propiedades.

Cuando hablamos de objetos físicos y de sus propiedades no igualamos 'consistente' y 'existente'. Ciertamente distinguimos, por

\footnotetext{
6 Una concepción de este tipo es la que subyace en la crítica de Strawson (p.48) al criterio ontológico de Quine cuando presenta al enunciado 'Hay al menos una propiedad que ninguna máquina posee, viz. la de eficiencia perfecta' como ejemplo de que al admitir su verdad admitimos la existencia de una propiedad no instanciada. Aunque al aceptar el postulado CPS no puedo aceptar esta crítica concreta creo, no obstante, que el clarificador análisis que ofrece Strawson en el lugar indicado, del problema de la existencia de propiedades, conduce a conclusiones afines a las nuestras.
} 
ejemplo, entre descripciones consistentes e inconsistentes de objetos físicos y si la descripción es inconsistente inferimos correctamente que no puede existir un objeto que la satisfaga (y esto último también resulta válido en los contextos puramente lógicos o matemáticos), pero de la consistencia de una descripción nunca deducimos la existencia del objeto físico al que pueda ajustar tal descripción. Y esto último es lo crucial.

Quizás nuestras últimas consideraciones hayan convencido al lector de algo que no pretendía, a saber, que la negación de CPS resulta tan injustificada como la negación de PS. Pero sea esto como sea lo que sí me parece claro es que la argumentación precedente cuenta como una razón decisiva para aceptar CPS, esto es, para proponer que adoptemos explícitamente CPS como un postulado, o regla, de significado a la par con PS y con los ejemplos anteriormente considerados de postulados de significado.

La conclusión de todo esto es clara: si consideramos justificada la aceptación de CPS junto con la de PS entonces hemos logrado justificar la paráfrasis ofrecida anteriormente a título de posibilidad: la expresión 'La propiedad P existe' no significa ni más ni menos que lo que signifique la expresión ' $\exists x$ Px' es verdadero'.

$X$. Nuestras consideraciones anteriores han mostrado en qué sentido cabe interpretar la expresión 'La propiedad $\mathrm{P}$ existe' como equivalente a la expresión ' $\exists x$ Px' es verdadero', y de aquí se sigue que en cuanto aceptemos un enunciado de la forma " $\exists \mathrm{x}$ Px' 
es verdadero' hemos aceptado con ello la existencia de al menos una propiedad.

Sin embargo hay una conocida objeción de tipo general a la admisión de la existencia de propiedades debida a Quine. Desde el punto de vista de este filósofo habría una asimetría entre la admisión de la existencia de objetos físicos y la admisión de propiedades. Tal asimetría sería consecuencia de que mientras que para los objetos físicos disponemos de un principio de individuación, a saber, dos objetos físicos son idénticos si y solo si son coextensos [TT,101], para propiedades carecemos de cualquier principio semejante. Más aún, incluso las clases concebidas como objetos abstractos poseen tal principio de individuación: dos clases son idénticas si y sólo si poseen los mismos miembros. En el caso de los objetos físicos la coextensividad es espacio-temporal, en el caso de los objetos abstractos del tipo de las clases la coextensividad es la de sus miembros: las clases mismas no están más individualizadas de lo que lo puedan estar sus elementos. Por eso es inútil para Quine intentar usar como principio de identificación de atributos (incluidas las propiedades, según su terminología) la definición: Dos atributos son idénticos si y solo si pertenecen a las mismas clases; pues estas clases tendrán como elementos atributos y por tanto quedarán tan carentes de identificación como estos últimos.

Tampoco funcionaría para Quine el intento de usar la coextensividad hacia abajo por así decir. Así dos propiedades serían idénticas si y sólo si cualquier objeto que tenga una de ellas tiene también la otra. Pero esto no funciona porque pese a la coextensividad de las propiedades criatura con riñones y criatura con corazón no diríamos que estamos ante la misma propiedad. 
XI. Bien, ¿qué decir de esta objeción? El núcleo de la objeción parece ser este: Los objetos tienen un principio de individuación, v.g. coextensividad, del que carecen las propiedades y en esta asimetría respecto a la posesión, o no, de un principio de individuación es en la que fundamenta la admisión ontológica de los objetos físicos, o abstractos como las clases, y el subsiguiente rechazo de las propiedades como entidades sospechosas.

Ahora bien, ¿está bien fundada esa asimetría? Lo estaría si la noción de coextensividad aplicada a objetos físicos por ejemplo, pudiera funcionar prescindiendo de cualquier apelación a las propiedades, y esto es precisamente lo que sugiero que no puede hacerse. Dicho brevemente: no podemos determinar si dos presuntos objetos son o no coextensos, v.g. si se trata o no del mismo objeto, sin recurrir a las propiedades poseídas por los objetos en cuestion.?

XII. Efectivamente ¿cómo identificamos a los objetos físicos paradigmáticos, los objetos macroscópicos?; o ¿cómo decidimos (por usar un ejemplo del propio Quine) si dos encuentros con una manzana son encuentros con manzanas diferentes oson dos encuentros con la misma manzana? Decir que se trata de la misma

\footnotetext{
7 El propio Quine viene a reconocer explícitamente en una ocasión que 'Las cosas son indistinguibles excepto por sus propiedades' [ OR,71]. Lástima que no sacara las consecuencias pertinentes de esta observación.
} 
manzana si los encuentros son con la misma región espaciotemporal no resuelve el problema porque ante este tipo de respuestas siempre cabe preguntar ¿Estamos o no ante la misma región espacio-temporal las dos veces?

Pero aún suponiendo resuelto el problema de la individuación de las regiones espacio-temporales, este tipo de problema se agudiza aún más cuando nos las habemos con propiedades copresentes en la misma región espacio-temporal. Así supongamos por un momento que todos y sólo los objetos rojos fueran esféricos. ¿Acaso no distinguiríamos en este hipotético caso entre la propiedad rojo y la propiedad esférico? ¿No distinguiríamos entre el color y la forma del objeto? En mi opinión la respuesta correcta a estas preguntas es clara, pero si se prefiere un ejemplo no contrafáctico (aunque formulado aún en forma subjuntiva) el siguiente podría servir: Supongamos que fabricamos un original objeto con una forma geométrica única -ningún otro objeto la posee- $\mathrm{y}$ con un material constituido por una nueva aleación desconocida hasta ese momento. ¿Continuaríamos manteniendo aún que ambas propiedades- correspondientes a forma y material- son indistinguibles por ser coextensas? $¿ \mathrm{O}$ admitiríamos que son distinguibles pero no les reconocemos existencia hasta tanto no dispongamos de un principio general de individuación?

El problema general en torno a la coextensividad, o falta de esta, entre dos propiedades es que si no hay algún modo de distinguirlas previamente no parece haber modo alguno de iniciar la investigación para determinar si son, o no, efectivamente coextensas. ¿Son coextensas las características propiedades del sabor, forma y textura de los limones? No parece haber forma de buscar una respuesta a esta pregunta sin distinguir previamente 
entre sí esas propiedades. Y ocurre que, de hecho, las distinguimos. ${ }^{8}$ Pero si las distinguimos iTiene algún sentido aún denegarles existencia? ${ }^{9}$

XIII. La presunta asimetría entre la existencia de objetos y la existencia de propiedades no tiene fundamento ontológico ni epistemológico. No tiene fundamento ontológico alguno porque los objetos no son nada más allá de las propiedades que poseen, ¿Acaso tiene algún sentido concebir objetos carentes de propiedades? Simétricamente: no habría propiedades si no existieran objetos que las instanciaran o ejemplificaran; y esto último no es más que una consecuencia directa de CPS.

Tampoco tiene fundamento epistemológico alguno la presunta asimetría entre objetos y propiedades porque para conocer un objeto se requiere conocer, al menos, alguna de las propiedades que posee (incluyendo entre las propiedades sus relaciones con otros objetos). ¿Tiene algún sentido decir que conocemos un objeto

8 Debo a Josefa Pardo el dato de que existe una variedad de limones que lejos de presentar el característico sabor agrio de los limones comunes poseen un sabor más bien dulce $y$, sin embargo, resultan indistinguibles del resto por su forma, color o textura.

9 Probablemente desde el punto de vista de Quine aún se podría intentar replicar que aunque para expecificar [specify] un objeto físico necesitamos apelar a sus propiedades, no es necesaria tal cosa para definir [define] su identidad [TT, 101]. Pero esta distinción se muestra ilusoria en cuanto pretendemos aplicarla a cualquier caso práctico de identificación de objetos como ilustran suficientemente los ejemplos examinados, y desde luego no parece afectar en modo alguno al argumento empleado por nosotros para demostrar como la verdad de enunciados formalizables de modo natura! en el lenguaje de la lógica de primer orden implica la existencia de propiedades. 
pero que desconocemos cualquiera de sus propiedades? Simétricamente: no es posible conocer propiedades si no somos capaces de reconocer sus instancias y distinguirlas de otros objetos que no las ejemplifican. ¿Tiene algun sentido decir que conocemos una propiedad pero que somos incapaces de reconocer cualquiera de sus instancias? ¿En qué consistiría entonces no conocer una propiedad?

Así en el sentido en el que proponemos aquí usar los términos 'objeto' y 'propiedad', los objetos no serían más que colecciones de propiedades copresentes.

¿Y las propiedades? Las propiedades serían aquello (sea lo que fuere) que hace verdaderos a algunos predicados, falsos a otros, y que permite identificar al objeto al tiempo que lo distingue de otros objetos.

Constituye un hecho cotidiano que efectuamos predicaciones de las cosas que nos rodean y que, en ocasiones, sólo tras una inspección minuciosa del objeto podemos decidir si nuestra predicación es verdadera o falsa del objeto en cuestión.

Así, por ejemplo, decimos que cierta prenda de vestir es impermeable al agua; y sólo después de un examen de su reacción cuando se vierte agua sobre ella podemos decidir con razonable seguridad si tal predicación era verdadera o falsa. Pues bien, la propiedad del objeto es precisamente aquello que hace que tal predicación resulte verdadera o falsa. Prescindir de la existencia de propiedades en las cosas dejaría en definitiva completamente inexplicado el hecho cotidiano de que ciertos predicados resultan aplicables a ciertas cosas pero no a otras; dejaría inexplicado el hecho de que a menudo -aunque no todo lo frecuente que sería de 
desear- corregimos nuestras iniciales predicaciones después de examinar más detenidamente los rasgos o propiedades de los objetos que describimos; dejaría inexplicado, en suma, el propio fenómeno del uso social del lenguaje.

Frente a tal negación de la existencia de propiedades, a menudo conjugada con la admisión de la existencia de objetos, nuestra propuesta se reduce a señalar la equivalencia entre hablar de la existencia de propiedades y hablar de la existencia de objetos; a considerar los objetos como colecciones de propiedades y a éstas como aquellos rasgos de los objetos que hacen verdaderos o falsos a los predicados. Esta es en definitiva, la concepción ontológica, o metafísica, que subyace en nuestra propuesta de considerar equivalentes las expresiones ' $\mathrm{La}$ propiedad $\mathrm{P}$ existe' y " $\exists \mathrm{x}$ Px' es verdadero'.

XIV. Se trata de una concepción metafísica mínima en el sentido de que resulta igualmente compatible con una concepción aristotélica, o con una concepción kantiana, de objetos y propiedades. Efectivamente, desde un punto de vista aristotélico podría decirse que la propiedad del objeto que hace verdadero a un enunciado de la forma ' $\exists x$ Px' es un rasgo que posee el objeto en sí mismo y que existe independientemente de nuestras peculiares estructuras cognoscitivas. Nada de lo que hemos dicho anteriormente excluye esta interpretación. Pero tampoco excluye una interpretación de tipo kantiano que arguyera que las propiedades que hacen verdaderas a ciertas predicaciones y falsas a otras no son rasgos de los objetos en sí mismos, sino rasgos, o propiedades, de 
los objetos tal como se muestran a seres dotados de ciertas estructuras perceptivas y cognoscitivas.

Aunque, repito, lo dicho anteriormente no excluye a ninguna de las dos interpretaciones mencionadas, creo no obstante que es la interpretación de corte kantiano la más aceptable pues no veo qué fundamento puede haber en la tesis de que las propiedades que tengan los objetos independientemente de como puedan aparecer a seres con determinadas estructuras perceptivas y cognoscitivas resulten ser idénticas a las propiedades concebidas por los seres con tales estructuras. Pero este es un grave problema que excede los límites del presente artículo. ${ }^{10}$

Agradezco a Jose Hierro sus interesantes y detalladas observaciones sobre el borrador de este articulo. 


\section{BIBLIOGRAFÍA}

ALEMAN, A., Teoria de las Categorias en la filosofía analítica. Tecnos, $2^{2}$ ed., 1996.

"Wittgenstein: Lógica, matemáticas y convención". Revista de Filosofía, vol. VIII, nº 14, 1995.

ARMSTRONG, D.M., Universals and Scientific Realism. Cambridge U.P., 1978 [J.A. Robles (trad.): Los Universales y el realismo cientifico. Universidad Autónoma de México, 1988; por donde cito].

CARNAP, R., Meaning and Neccessity. University of Chicago Press, 1947, 1970.

QUINE, W.v.O., [OR] Ontological Relativity \& Other Essays. Columbia University Press, 1969.

[WP] The Ways of Paradox and Other Essays. Harvard University Press, 1976.

, [TT] Theories and Things. Harvard U. P., 1981.

, "Respuesta a Blasco", en M. Garrido y otros: Aspectos de la filosofia de W. V. Quine. Teorema, 1975.

SCHEFFLER, Y. y CHOMSKY, N., "What Is Said To Be". Meeting of the Aristotelian Society on $24^{\text {th }}$ November, 1958.

STRAWSON, P., Analysis and Metaphysics. Oxford U.P., 1992. 